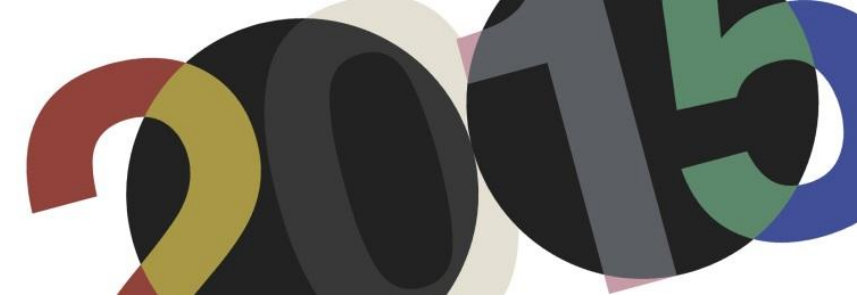

DOI: http://dx.doi.org/10.4995/LC2015.2015.664

\title{
Georges Candilis (1913-1995) architecte pour le plus grand nombre
}

\author{
L.J. Duport
}

Ecole Nationale Supérieure d'Architecture de Montpellier

Résumé: Né à Bakou en 1913 Georges Candilis est un architecte d'origine grecque qui étudie à l'Ecole Supérieure Polytechnique d'Athènes où il rencontre Le Corbusier en 1933 lors du 4e congrès des CIAM. Arrivé à Paris en 1945 il intègre l'Atelier de Le Corbusier où il travaille exclusivement sur les études et le chantier de l'Unité d'Habitation de Marseille. Après avoir été chargé de représenter Le Corbusier au 7e CIAM à Bergamo en 1949 Candilis va se rendre au Maroc où il va construire des nombreuses opérations en qualité de directeur de l'ATBAT Afrique et de membre du groupe GAMMA. Il va se révéler un des acteurs du Team X assurant le passage des CIAM au Team X dont il organisera 5 réunions entre 1960 et 1977. De retour en France en 1955 Candilis va s'associer avec les architectes Woods et Josic. L'équipe va remporter le concours Million et construire près de 4000 logements à Bagnols sur Cèze, Toulouse et en région parisienne. D'autres concours vont suivre en France et à l'étranger, l'équipe construira ainsi l'université libre de Berlin. Par la suite Candilis assure seul la mission d'architecte en chef de la station de Leucate Barcares (1962-1976) et entre 1970 et 1978 il est chargé de plusieurs projets au Moyen Orient. Parallèlement Candilis est impliqué dans la diffusion de l'architecture dès 1953 comme membre du comité de rédaction de revues et dans l'enseignement en qualité de professeur à partir de 1963. Il s'éteint à Paris le 10 mai 1995.

Abstract: Born in Baku in 1913 Georges Candilis is an architect of Greek origin who studied at the Polytechnic School of Athens where he met Le Corbusier in 1933 at the 4th Congress of CIAM. Arrived in Paris in 1945 he joined the Atelier of Le Corbusier where he works exclusively on studies and the site of the Unité d'habitation in Marseilles. After being appointed to represent Le Corbusier at the 7th CIAM in Bergamo in 1949 Candilis will travel to Morocco where he will build many operations as Director of ATBAT Africa and a member of the GAMMA group. It will be one of the actors of Team X and ensure the transition from CIAM to Team X for which he will hold 5 meetings between 1960 and 1977. Back in France in 1955 Candilis will partner with architects Alexis Josic and Shadrach Woods. The team will win the Million competition and build nearly 4,000 housing units in Bagnols sur Cèze, Toulouse and around Paris. Other competitions will follow in France and abroad, the team will thus build the Free University in Berlin. Subsequently Candilis assumes alone the chief architect mission of Leucate Barcares station (1962-1976) and between 1970 and 1978 he was responsible for several projects in the Middle East. In parrallel Candilis is involved in the diffusion of architecture since 1953 as an editorial board member of reviews and in architectural education with a grade of Professor since 1963. He died in Paris on May 10, 1995.

Mots-clés: CIAM, Team X, Enseignement, Habitat, Tige, Web.

Keywords: CIAM, Team X, Education, Housing, Stem, Web. 
Parallèlement à la célébration du cinquantenaire de la disparition de Le Corbusier, l'année 2015 est également l'occasion de commémorer le 20e anniversaire de celle de Georges Candilis.

Né à Bakou (capitale de Azerbaïdjan) le 29 mars 1913 (ou le 11 avril 1913 selon les sources) Georges Candilis s'éteint à Paris le 10 mai 1995. Avec ses parents il sillonne la Russie (Bakou, Rostov, Moscou) en étant le témoin de nombreux événements comme "les massacres entre Arméniens et Tartares, la guerre civile entre rouges et blancs et le triomphe des premiers ${ }^{1} \gg$ puis se rend en Turquie avant d'exiler et d'arriver en Grèce à l'âge de 12 ans, obtenant en 1926 la nationalité Grecque.

A la manière d'un choix qui réalise la synthèse entre le monde artistique de sa mère, qui était professeur de piano (comme la mère de Le Corbusier) et celui de son père, commerçant, il intègre en 1931 l'Ecole Supérieure Polytechnique d'Athènes, où il obtient son diplôme d'architecte et ingénieur en 1936. De cet enseignement Candilis garde le souvenir de deux professeurs exceptionnels Dimitri Pikionis ${ }^{2}$ et Anastase Orlandos ${ }^{3}$, «l'un m'a enseigné à comprendre, l'autre à connaître. L'enseignement de l'un sans l'autre n'aurait jamais été la même chose $»^{4}$.

Mais c'est surtout là où il rencontre Le Corbusier à l'occasion d'un événement majeur dont la portée et l'influence vont être déterminants pour lui : le 4e Congrès des CIAM de $1933^{5}$ qui se déroule non seulement à Athènes mais dans l'Ecole Polytechnique même. Au programme : la question des villes et de l'urbanisme comme «prolongement d'attitudes architecturales à l'échelle sociale ». Officiellement le Congrès ne commence qu'à Athènes avec l'inauguration d'une exposition de trente-trois plans de villes analysées et augmentés de photographies de réalisations architecturales de membres du Congrès. Mais celui-ci a en fait démarré sur le SS Patris II où la personne de Le Corbusier est omniprésente comme en témoignent certains participants et surtout le film « Architects Congress » de Lazlo Moholy-Nagy ${ }^{7}$.

Georges Candilis assiste à la conférence intitulée « Air-Son-Lumière » donnée par Le Corbusier que tout le monde attend et où ce dernier, arrivé en retard, rend un hommage à l'Acropole en introduction puis présente sa conception de l'immeuble d'habitation équipé de façades-rideaux tempérées. A la fin du congrès, enthousiaste, Candilis avec d'autres architectes et étudiants participe à la création de la section grecque des CIAM. A l'exception du VIe Congrès à Bergame en 1947, il sera désormais impliqué dans la totalité des congrès des CIAM d'après-guerre qui vont se succéder jusqu'en 1959.

De 1936 à 1940 il enseigne à l'École polytechnique d'Athènes puis à l'École nationale Sivitanivios pour la formation des techniciens du bâtiment (1940-1945) il est architecte du ministère des Travaux publics, puis exerce en libéral, construisant de modestes réalisations. Le climat politique qui régnait en Grèce avec la mise en place d'une république dès 1928 et surtout la montée en puissance du général Metaxas ${ }^{8}$, pousse Candilis et quelques autres à une opposition violente, à une prise de conscience et à un engagement politique, puis militaire.

\footnotetext{
${ }^{1}$ Demetriades Dimitri \& Papadaniel Dimitri Entretiens avec Georges Candilis, Article de la revue des Ingénieurs et architectes suisses, 1994.

2 Dimitri Pikionis (1887-1968) architecte et protagoniste grec des CIAM, développe une conception de l'intégration de l'architecture moderne dans le paysage en parfaite continuité avec la culture méditerranéenne.

${ }^{3}$ Anastase Orlandos (1887-1979) architecte et docteur ès lettres.

${ }^{4}$ Demetriades Dimitri \& Papadaniel Dimitri Entretiens avec Georges Candilis, Article de la revue des Ingénieurs et architectes suisses, 1994

${ }^{5}$ CIAM 4 Athènes 29 juillet-13 août 1933

${ }^{6}$ Candilis Georges: Bâtir la vie, un architecte témoin de son temps, Paris: Stock, 1977 p.82

${ }^{7}$ La copie du film est conservée à l'Institut gta, EPF Zurich.

${ }^{8}$ Ioannis Metaxas (1871-1941) général et homme politique, dictateur de la Grèce de 1936 à 1941
} 


\section{Chez Le Corbusier}

A la fin de la guerre en 1945, grâce à une bourse du gouvernement français Georges Candilis se rend à Paris et après une courte expérience chez André Lurçat, il se présente à l'Atelier du 35 rue de Sèvres où il est reçu par Le Corbusier en personne. Ce dernier l'engage pour s'occuper de l'Unité d'Habitation de Marseille, projet sur lequel il travaillera exclusivement. Dans l'équipe Georges Candilis côtoie Roger Aujame, Fernand Gardien, Jerzy Soltan André Wogensky et surtout Shadrach Woods ${ }^{9}$ avec qui il s'associera plus tard. Il prend conscience de la préoccupation architecturale d'après-guerre : la reconstruction massive de logements avec une administration française qui établit des normes et un cadre réglementaire rigide par la détermination de logements types, de surfaces minimum, l'utilisation de standards rationalisant la construction : portes, fenêtres, placards, planchers.

Mais l'Unité d'Habitation est un projet sur lequel Le Corbusier travaille depuis de longues années et celle de Marseille se révèle un prototype où il met en œuvre une grande partie de ses théories avec l'application du modulor, de la polychromie et la mise en place à l'échelle de trois cent cinquante logements de principes expérimentés auparavant comme les pilotis, la toiture terrasse, les brise-soleil, les pans de verre. Georges Candilis sera chargé des études et de suivre le chantier en relation avec Vladimir Bodiansky ${ }^{10}$ et l'ATBAT ${ }^{11}$. Cette expérience où architecture et ingénierie sont étroitement liées, où la conception du projet est immédiatement suivie de sa réalisation, lui permet de rencontrer à l'atelier de Le Corbusier ou sur le chantier, des personnalités politiques, des artistes, de nombreux architectes et critiques impliqués sur la scène architecturale française et internationale, notamment dans les CIAM. Il sera même chargé de représenter Le Corbusier au 7e CIAM à Bergamo du 23 au 31 juillet $1949^{12}$. Cette participation se traduit par la présentation d'un panneau/dépliant explicatif de l'Unité d'habitation de Marseille réalisé par l'ensemble du groupe Ascoral ${ }^{13}$ des Jeunes dont Candilis est le responsable du comité de rédaction.

Le dépliant qui se veut l'application de la grille CIAM pour la mise en pratique de la Charte d'Athènes explique minutieusement la conception et présente des photos des différentes étapes de la construction de l'unité de grandeur conforme. "J'installe donc le logis au cœur du binôme " individuel + collectif », et la liberté individuelle étant assurée par le logis, j'organise tout ce que le collectif peut apporter $»^{14}$

\footnotetext{
${ }^{9}$ Schadrach Woods (Yonkers 1923- New York 1973) est un architecte, urbaniste et théoricien américain qui travaille chez Le Corbusier en 1948, puis à partir de 1951 avec Georges Candilis au sein de l'ATBAT Afrique. Ils sont rejoints, de retour à Paris en 1954 par Alexis Josic (1921-2011). En 1970 il s'installe aux Etats Unis où il exerce et enseigne jusqu'en 1973

${ }^{10}$ Vladimir Bodiansky (1894-1966) est un ingénieur français d'origine russe qui crée l'ATBAT en 1945 avec Le Corbusier puis l'ATBAT Afrique en 1951 avec Georges Candilis

${ }^{11}$ A.T.B.A.T Atelier des bâtisseurs sera le bureau d'études techniques en charge de la construction de l'Unité d'Habitation de Marseille sous la direction de Bodiansky voir les articles dans Architectural Design, Janvier 1965

${ }^{12}$ Voir lettre de Le Corbusier à Candilis datée du 24 Mai 1949, archives Famille Candilis

${ }^{13}$ L'Assemblée de Constructeurs pour une Rénovation architecturale fondée à Paris en 1943 par Le Corbusier, visait à établir les modalités pour joindre la reconstruction à l'introduction de préfabrication et industrialisation de la construction

${ }^{14}$ Le Corbusier in dépliant de présentation au $7^{\mathrm{e}}$ congrès CIAM à Bergame, Special Collections, Frances Loeb Library / Harvard University GSD
} 

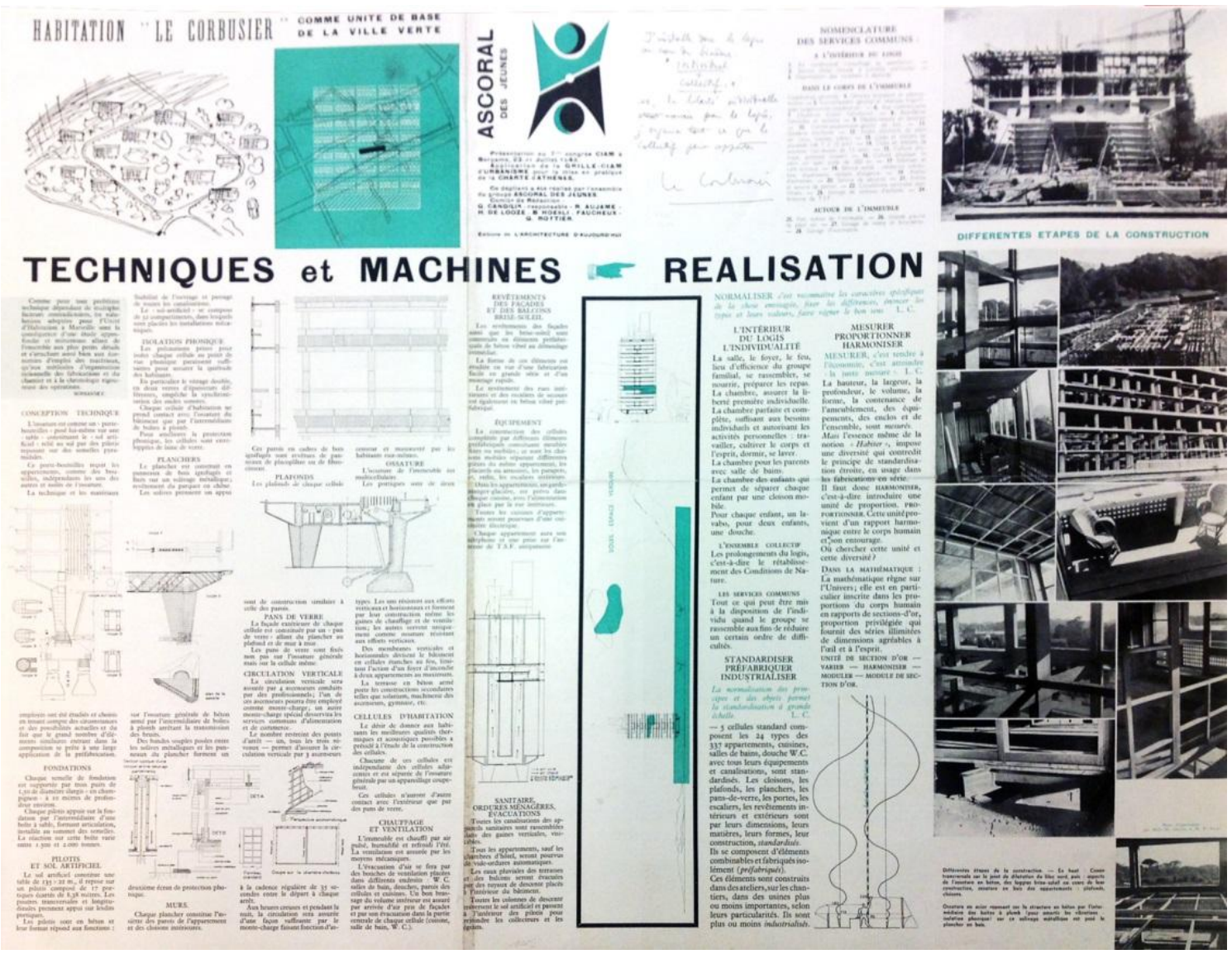

1. Dépliant de présentation pour le 7e congrès CIAM à Bergame. OFLC-ADAGP

A la fin du chantier Georges Candilis considérant que le rôle qu'il avait tenu auprès de Le Corbusier était terminé, décline la proposition qui lui est faîte de suivre le chantier de Chandigarh aux côtés de Pierre Jeanneret mais accepte celle de Bodiansky de devenir le directeur de la filiale de l'ATBAT au Maroc, l'ATBAT-Afrique et choisit de " se tourner vers des régions vierges et de se pencher sur l'architecture du Plus Grand Nombre ${ }^{15}$.A partir de 1951, cet outil de travail permet à Georges Candilis et Shadrach Woods de construire une cité expérimentale de 150 logements pour la population musulmane à Casablanca (Carrières Centrales 1953), puis pour la population européenne à Oran (1954-1955) et à Sidi Bel Abbès (1954).

\footnotetext{
${ }^{15}$ Bâtir la vie, op. cit. p.182
} 

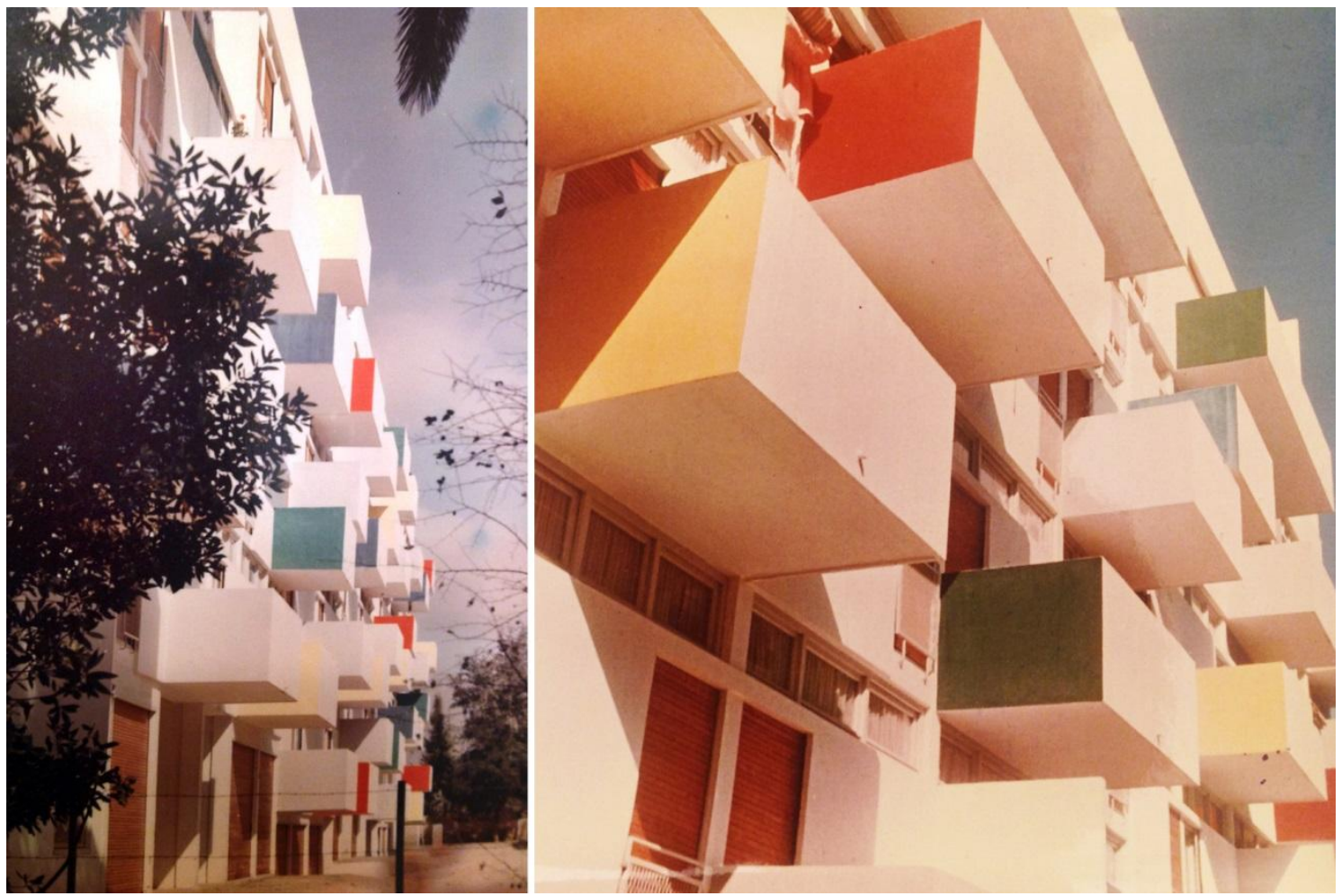

2. Immeuble à Sidi Bel Abbès, Candilis \& Woods architectes, 1954

Pour Candilis et Woods il s'agissait de trouver des formes architecturales, des structures qui tenaient compte des conditions économiques et sociales des plus démunis en leur apportant un élément essentiel dont ils étaient privés : la dignité.

Afin de remplacer les bidonvilles Michel Ecochard ${ }^{16}$ avait conçu des logements simples et économiques, un carré de $8 \mathrm{~m}$ par $8 \mathrm{~m}$ comportant deux chambres, une cour et un petit sanitaire, d'une surface totale de $64 \mathrm{~m}^{2}$. Fondés sur l'analyse des médinas anciennes ces logements étaient assemblés à rez-de-chaussée dans une trame urbaine, constructible en grande série, permettant préfabrication, industrialisation et de loger 350 habitants à l'hectare. Sur ce principe, et avec l'accord d'Ecochard Candilis et Woods proposent, des assemblages sur quatre ou cinq niveaux dans l'idée de réduire par la densité les zones étendues des bidonvilles et de limiter la spéculation foncière en s'appuyant sur une nouvelle interprétation de l'habitation collective marocaine avec des recherches sur trame modulor ${ }^{17}$.

\footnotetext{
${ }^{16}$ Michel Ecochard (1905-1985) architecte et urbaniste français en charge de Casablanca et travaille au Pakistan, au Liban et en Syrie.

${ }^{17}$ Candilis Georges \& Woods Shadrach photographie dessin sur calque Habitation collective marocaine, recherches sur la trame Modulor, Déc.1951, Woods Archives, Avery Architectural and Fine arts Library, Columbia University
} 


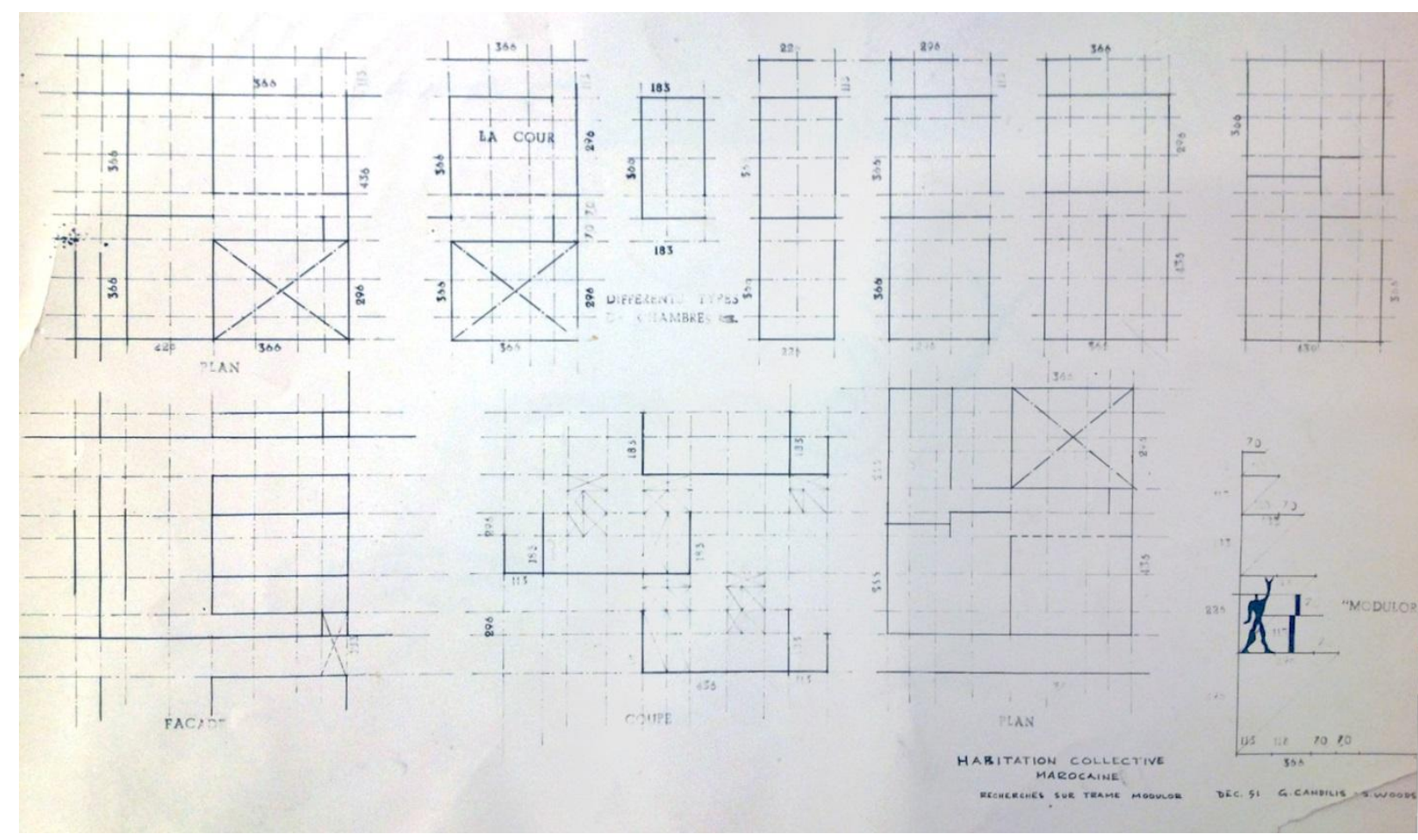

3. Candilis Georges \& Woods Shadrach Habitation collective marocaine, recherches sur la trame Modulor,1951.

La maison musulmane traditionnelle est ainsi prise comme modèle avec une série de pièces groupées autour d'une cour intérieure à ciel ouvert, cette dernière assurant l'éclairage et la ventilation des pièces et étant utilisée comme espace à fonctions multiple.Avec l'appui d'Ecochard Candilis et Woods réussissent en 1953 avec Bodiansky à construire une cité expérimentale de 150 logements dans les Carrières Centrales de Casablanca, au milieu des maisons à patio en développant trois types d'immeubles selon deux facteurs : l'ensoleillement et le niveau d'évolution des habitants.Ces réalisations réinterprètent les projets des constructivistes russes de la fin des années des années 1920 et notamment les projets de Moisei Ginzbourg ${ }^{18}$ dont Candilis connait les réalisations à travers les publications et notamment la revue de l'association OSA СОВРЕМЕННАЯ АРХИТЕКТҮРА, l'Architecture Contemporaine ${ }^{19}$.

\footnotetext{
${ }^{18}$ Moisei Ginzbourg (1892-1946) architecte constructiviste auteur de l'immeuble Narkomfin à Moscou qui aura une grande influence chez de nombreux architectes notamment grâce à la coupe

${ }^{19}$ Revue СОВРЕМЕННАЯ АРХИТЕКТҮРА, l'Architecture contemporaine nº 4 \& 5, Р. 130 à 145 , archives Famille Candilis
} 

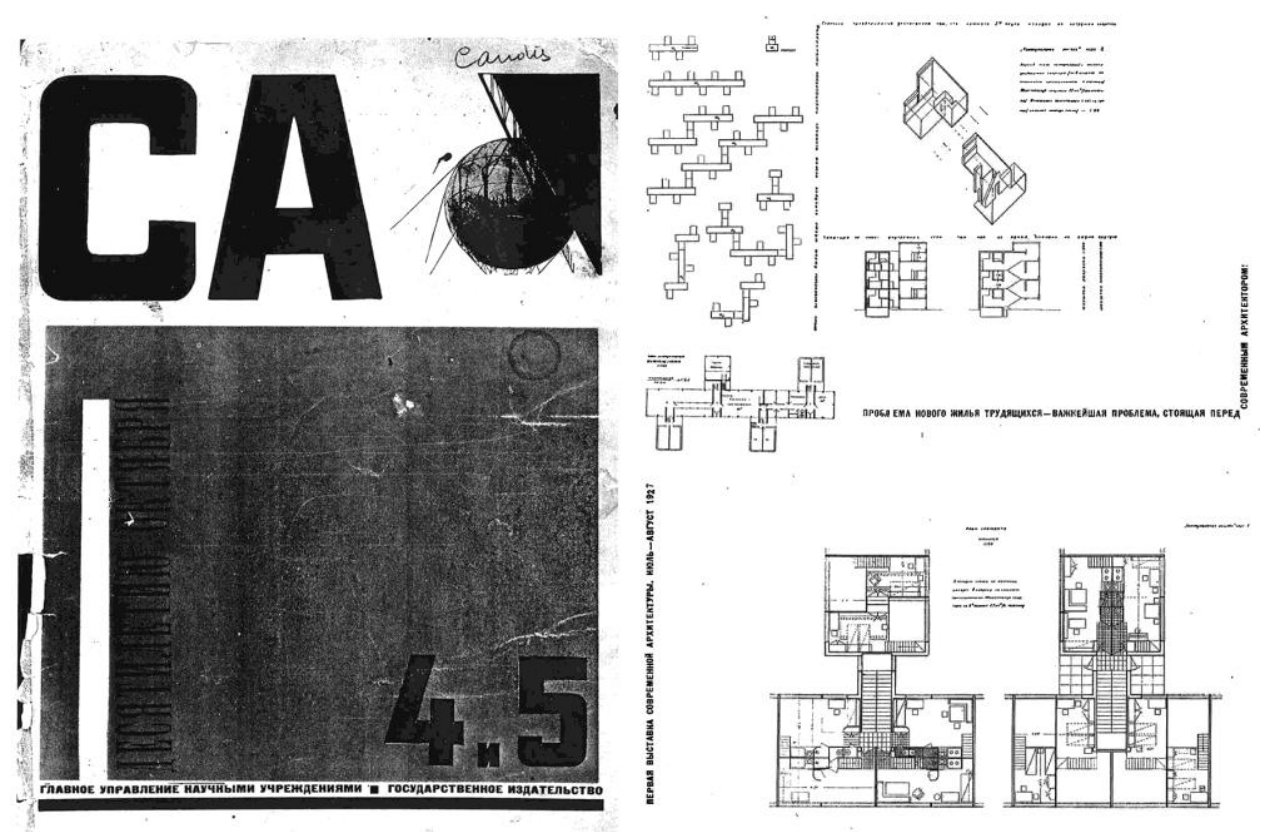

4. Revue СОВРЕМЕННАЯ АРХИТЕКТҮРА, l’Architecture contemporaine $n^{\circ} 4 \& 5$

Ainsi sont conçus les immeubles d'habitation « Nid d'Abeilles » aux superpositions simples et idéalement éclairées et l'immeuble "Sémiramis » partagé en deux pour suivre la topographie introduisant ainsi de nouvelles typologies qui seront présentées en 1953 au C.I.A.M.9 d'Aix en Provence par le groupe GAMMA dirigé par Georges Candilis. Ce projet est largement publié et fera notamment la couverture du numéro 57 de décembre 1954 de l'Architecture d'Aujourd'hui dont Candilis est membre du comité de rédaction depuis 1953 et pendant plus de 20 ans. Par la suite ce projet sera à nouveau mis en avant notamment dans un article par Alison \& Peter Smithson qui voient là « l'une des plus grande réalisations depuis l'Unité d'Habitation de Le Corbusier à Marseille $»^{20}$.

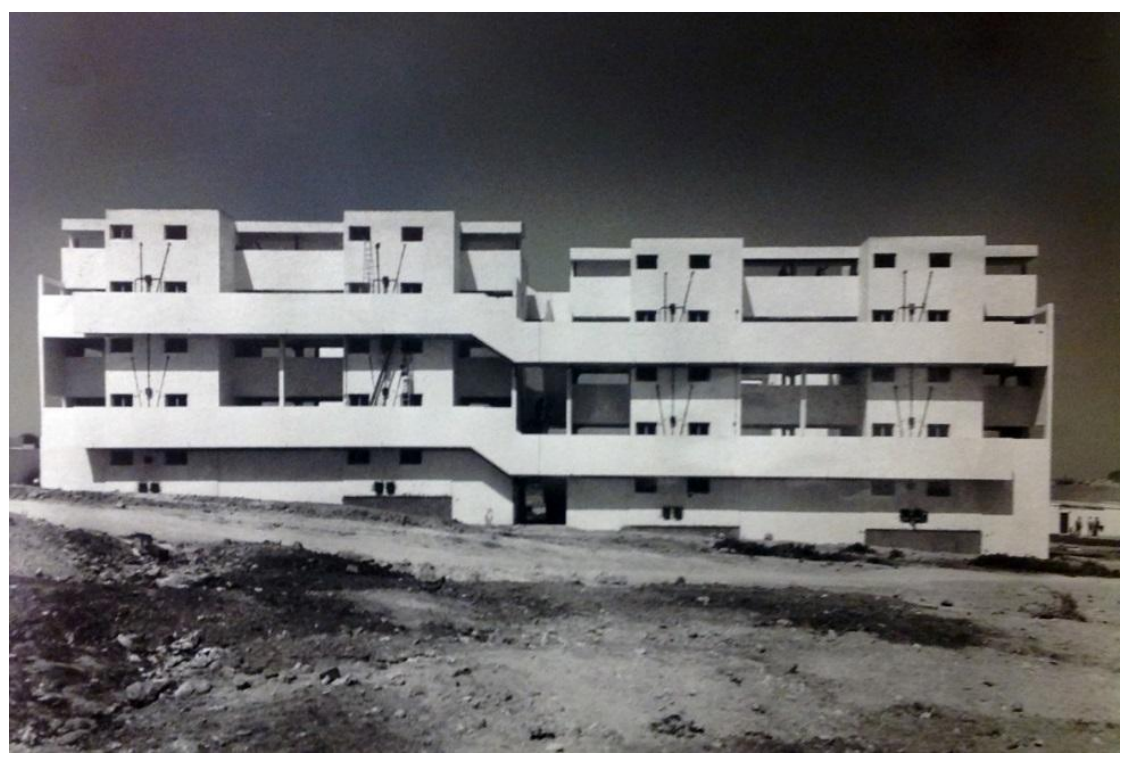

5. Immeuble Sémiramis, Carrières Centrales, Casablanca, Candilis \& Woods architectes.

\footnotetext{
${ }^{20}$ Smithson Alison \& Peter Architectural Design, 1955
} 


\section{Le groupe GAMMA}

La création du groupe GAMMA (Groupe d'Architectes Modernes Marocains) est le résultat d'actions convergentes et successives de Michel Ecochard et de Georges Candilis ${ }^{21}$. Sigfried Giedion ${ }^{22}$ avait demandé à Ecochard en 1947 d'organiser un voyage au Maroc et une contribution au CIAM. L'arrivée de Candilis au Maroc comme directeur de l'Atbat Afrique permet à la fois une réconciliation entre le groupe d'Ecochard et le département de planification urbaine du service de l'urbanisme du Maroc et sa nomination comme animateur du groupe GAMMA auprès des CIAM ${ }^{23}$.

\subsection{Georges Candilis et les CIAM}

Il est nécessaire de revenir sur l'influence de Georges Candilis dans la préparation du CIAM 9 d'Aix en Provence. A l'invitation du groupe suédois des CIAM, une grande réunion a lieu à Sigtuna, près de Stockholm du 25 au 30 juin 1952, quelques temps après une première réunion à Paris chez Le Corbusier.

A Sigtuna, Georges Candilis dirige la 6e et dernière session sur la place de la jeune génération dans les groupes des CIAM. Il commence la discussion avec un manifeste que l'on peut considérer comme la genèse de Team Ten, indiquant qu'il avait noté à Bergame la présence de deux familles : celle qui a fondé l'architecture moderne et celle qui a travaillé sur les bases prévues par les fondateurs.

Le CIAM 9 doit selon lui «marquer une frontière entre deux phases de travaux des CIAM et en même temps entre deux générations ». Il s'appuie en cela sur la conclusion de la réunion de Paris où une lettre co-signée par Le Corbusier, Sigfried Giedion et Jacqueline Tyrwhitt ${ }^{24}$ indique que, concernant le futur des CIAM il serait bon de prévoir une période de transition jusqu'au CIAM 10, que durant cette période une attention particulière pourrait être donnée au CIAM 9 aux travaux de jeunes groupes, et que le thème et l'organisation du CIAM 10 serait placés dans les mains d'une nouvelle génération d'architectes. La circulaire concluant qu'à la lumière du travail montré lors du CIAM 10, le congrès pourrait alors décider si la nouvelle génération a le talent et la vitalité de porter les travaux des CIAM.

Parallèlement à cette discussion sur les anciens et la jeune génération, Candilis insiste pour que le thème du CIAM 9 porte sur l'habitat et non sur le logement, sujet déjà traité lors de plusieurs congrès précédents à Francfort, Bruxelles, Paris, en argumentant qu'une charte de l'habitat serait aussi importante que les résultats du CIAM d'Athènes ${ }^{25}$.

La notion d'habitat est si nouvelle dans le milieu architectural anglo-saxon que les Smithson trouvent nécessaire de le définir avec l'exemple montré par Candilis indiquant que « Habitat est le mot qu'utilisent les français pour décrire non seulement la maison mais aussi son environnement et tout ce qui en relève $»^{26}$.

Mais c'est surtout au CIAM 9 que GAMMA présente ses travaux et tente de lier les résultats du « logement pour le plus grand nombre » à la charte de l'habitat proposée. Le projet présenté par Candilis et Woods pour les

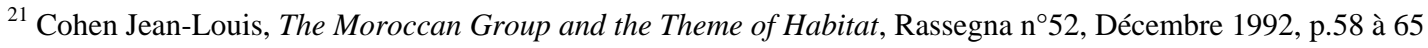

${ }^{22}$ Siegfried Giedion (1888-1968), historien et critique de l'architecture, professeur à Harvard et MIT, secrétaire général des CIAM

${ }^{23}$ Cohen Jean-Louis ; Eleb Monique: Casablanca : Mythes et figures d'une aventure urbaine, Paris: Hazan, 1998

${ }^{24}$ Jaquelyne Tyrwhitt (1905-1983), paysagiste et urbaniste, professeur à Harvard et membre des CIAM

${ }^{25}$ Mumford Eric: The CIAM discourse on urbanism 1928-1960, Cambridge: MIT Press, 2000, p.255 et Sarkis Hashim: CASE : Le Corbusier's Venice Hospital and the Mat Building Revival, Munich-London-New York: Prestel Verlag, 2001, p.50 à 53

${ }^{26}$ Alison et Peter Smithson "Collective Housing in Morocco" Architectural Design, janvier 1955, cité par Monique Eleb, à l'universalisme fonctionnaliste, Ecochard, Candilis et l'ATBAT-Afrique, Les Cahiers de la recherche architecturale et urbaine, ${ }^{\circ} 2-3,1999$
} 
Carrières Centrales à Casablanca va constituer une étape importante dans la première manifestation « d'une nouvelle manière de penser» ${ }^{27}$.

A la fin du CIAM 9 à Aix en Provence, un petit groupe est formé avec Georges Candilis \& Shadrach Woods pour la France, Jaap Bakema et Aldo Van Eyck pour la Hollande, Rolf Gutmann pour la Suisse, Alison et Peter Smithson, Bill Howell et John Voelker pour l'Angleterre. C'est ce groupe dit des « jeunes » qui sera chargé de l’organisation du CIAM X à Dubrovnik en 1956 et allait devenir le Team pour le Ten ou encore Team X.

\subsection{L'équipe Candilis/Josic/Woods}

De retour à Paris en 1955, Georges Candilis et Shadrach Woods s'associent avec l'architecte Alexis Josic. L'équipe propose une réflexion architecturale nouvelle centrée sur l'habitat. Ainsi pour Georges Candilis, l'habitat est le lieu de l'universel et du contingent. Habiter c'est répondre, d'une part aux besoins universels de se réunir et de s'isoler, et, d'autre part, aux conditions particulières du lieu.

« Inventer des logements simples, véritablement économiques, qui possèdent la qualité du respect, ce n’était pas de la grande architecture, mais cela représentait autant de difficultés, et demandait plus d'imagination et de sensibilité que de construire des palais $»^{28}$.

L'équipe Candilis/Josic/Woods va accompagner ses conceptions de publications : « Depuis toujours, l'homme lutte pour créer son foyer. C'est un des efforts les plus vitaux de son existence. En suivant les différentes phases de cette lutte, nous traçons les grandes lignes de notre conception. 1. recherche d'un lieu 2. Protection contre le froid.3.Équipement vital : eau, lumière, chaleur, évacuation.4. Détermination des fonctions de base : s'isoler, se réunir. Dès sa création, le foyer est constamment évolutif. Le logement doit évoluer également en s'adaptant aux nécessités nouvelles ${ }^{29}$.

L'évolution de cette réflexion va concerner l'organisation urbaine et en particulier l'intégration du logement dans l'espace public : la continuité urbaine va être inventée ou réinventée jusqu'à la constitution de nappes continues. Ce processus va amener l'équipe à contester la charte d'Athènes qui «...avait provoqué l'éclosion de bâtiments, grands et petits, isolés, répétés à l'infini. Au lieu d'aligner des blocs nous avons cherché à les articuler entre eux, à les relayer, à créer des espaces indéfinissables qui rompent avec la monotonie et rendent une échelle agréable. A ce moment déjà c'était lutter contre le gigantisme et humaniser l'habitat ${ }^{30}$

A l'expérience marocaine de l'ATBAT Afrique s'ajoute celle du concours Million dont ils sont lauréats en 1955. L'équipe propose une réflexion architecturale avec l'opération Million, campagne du gouvernement français pour abaisser le coût de construction d'un logement de 3 pièces de $48 \mathrm{~m}^{2}$ pour un budget d'1 million de Francs de l'époque en élaborant des modèles standards applicables immédiatement. Avec l'opération Million on change l'échelle qui transforme les opérations en grands ensembles non plus selon une composition classique de plan de masse mais suivant d'autres dispositions

L'équipe Candilis-Josic-Woods réalisera ainsi de près de 4000 logements en région parisienne : logements collectifs à Ivry, Cité Emmaüs à Blanc-Mesnil (1955-1957), Bobigny (1956-1962) et Gennevilliers (1955-1956). L'équipe est également chargée du projet d'extension de la Ville de Bagnols sur Cèze qui vaudra à cette réalisation le Grand Prix National d'Urbanisme en 1959.

\footnotetext{
${ }^{27}$ Smithson Alison et Peter "Collective Housing in Morocco" Architectural Design, janvier 1955

${ }^{28}$ Candilis Georges: Bâtir la vie, un architecte témoin de son temps, Paris: Stock, 1977 p.185

${ }^{29}$ Candilis G., Josic A., Woods S., « Proposition pour un habitat évolutif » Techniques et Architecture n², 1959 pp.82-85

${ }^{30}$ Candilis Georges: Bâtir la vie, un architecte témoin de son temps, Paris: Stock, 1977 p.38
} 


\section{Bagnols sur Cèze}

La construction en 1954 de la première usine de production d'énergie nucléaire de la rive droite du Rhône à Marcoule (Gard) posait la question de l'habitat de nombreux arrivants amenés à travailler sur le site. Le commissariat à l'Energie Atomique et le Ministère de la Construction décident alors la création d'un « véritable et nouveau centre urbain » sur la commune de Bagnols sur Cèze, à une dizaine de kilomètres de Marcoule qui voit sa population passer de 5000 habitants (en 1956) à plus de 18000 habitants. Bagnols sur Cèze est une ville romaine située sur un carrefour de la route Nîmes-Lyon, c'est une cité médiévale fortifiée, un carrefour routier important et le centre commercial et administratif d'une région agricole. En 1956 Georges Candilis est contacté par Auguste Mionne ${ }^{31}$ le directeur de la Construction Moderne Française, Ce dernier a vu Candilis à l'œuvre quelques années plus tôt, quand il suivait le chantier de l'unité d'habitation de Marseille pour Le Corbusier. Auguste Mionne, mandaté par le Commissariat à l'Energie Atomique pour la construction de logements pour les ouvriers, les cadres et techniciens supérieurs, demande à Candilis de construire quatre-vingt logements en six mois.

\subsection{Le site :}

La topographie et le climat avec les crues de la Cèze et le Mistral au Nord, la route nationale à l'Ouest et la voie ferrée à l'Est imposent l'extension de la ville vers le Sud.

A cela s'ajoutent principes de préservations des traces historiques du paysage : arbres, anciens puits, ancienne muraille, un vallonnement naturel, «la présence des vestiges d'un ancien théâtre romain nous incita à favoriser cette percée nouvelle vers le sud. C'est dans cette direction que nous avons envisagé les prolongements de la cité» $^{32}$

\subsection{La composition :}

Le premier acte a été la création d'un élément de liaison entre la vieille ville et la nouvelle afin d'éviter la ségrégation en positionnant le centre commercial et le centre culturel à la croisée des deux villes. La nouvelle ville a été directement inspirée de l'ancienne, ainsi les rues ne sont pas à angles droits et les unités de logements projetées forment des redents évitant l'alignement en ligne droite. De même trois points dominent Bagnols sur Cèze et servent de repères : une tour romane et deux clochers gothiques. L'extension nouvelle reprend « en contrepoint le thème de ces trois pôles et implante résolument parmi des bâtiments de un ou cinq niveaux, six (deux fois trois) tours de quinze niveaux $»^{33}$.

\footnotetext{
${ }^{31}$ Auguste Mionne (1898-1962) est un entrepreneur créateur en 1936 de la Construction Moderne Française, une Société Coopérative qui sera en charge de l'Unité d'Habitation de Marseille (arch. Le Corbusier), des opérations de logements à Bagnols sur Cèze, Nîmes, Marseille, Béziers (arch. Candilis/Josic/Woods)

${ }^{32}$ Candilis Georges: Bâtir la vie, un architecte témoin de son temps, Paris: Stock, 1977 p.214

${ }^{33}$ Voir la brochure Urbanisme en France du Ministère de la Construction « Bagnols sur Cèze »
} 

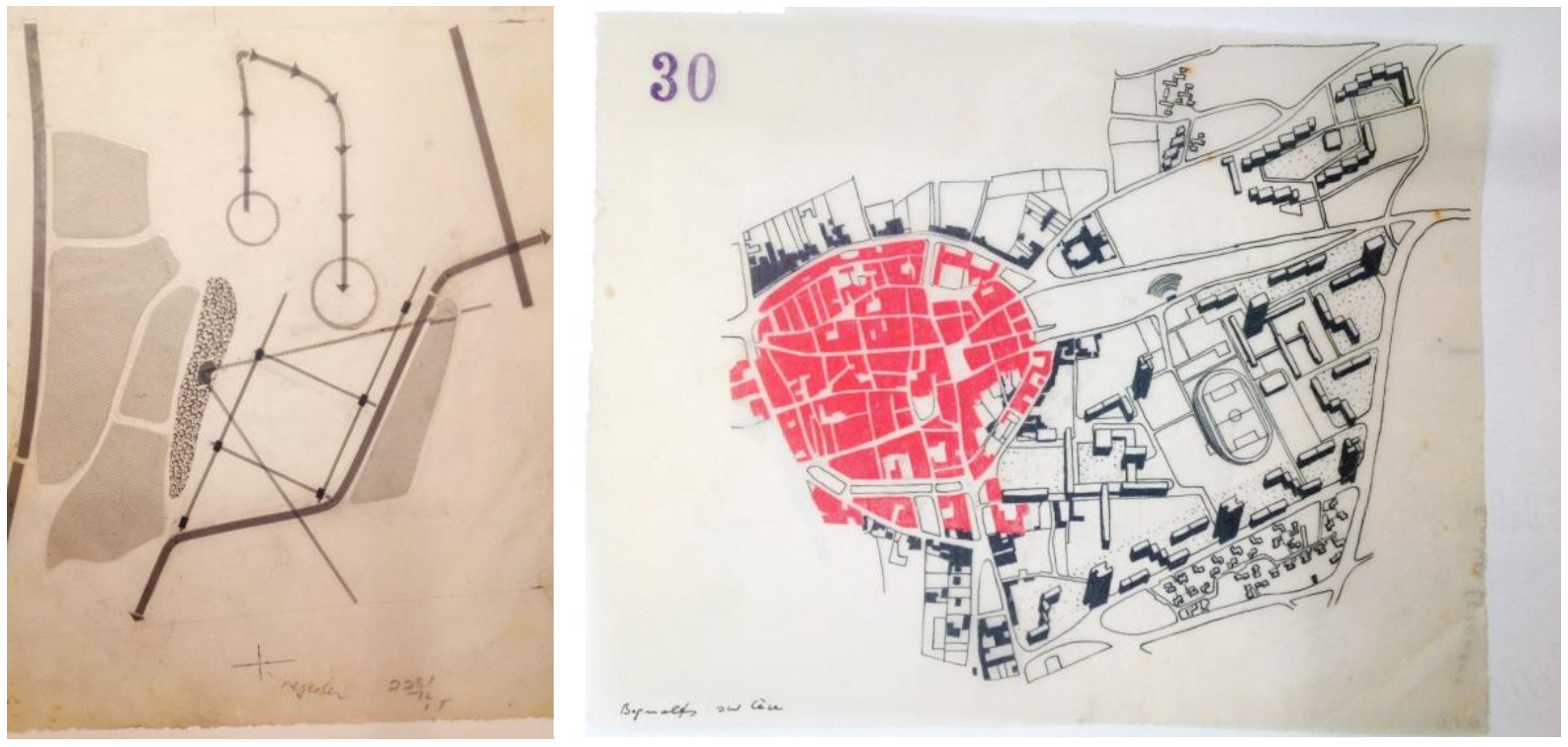

6. Croquis et schémas d'intention, extension de Bagnols sur Cèze, Candilis/Josic/Woods

L'opération distingue quatre entités : le Quartier de la Coronnelle (un premier quartier réalisé dans l'urgence), le Quartier de la Citadelle comprenant 370 logements, un centre commercial et une école de quartier construits en onze mois, période permettant l'étude de l'extension de la ville, le Quartier des Escanaux, qui constitue la principale extension de la ville avec la construction de 1200 logements et enfin la cité-jardin « Le Bosquet », un ensemble de 30 villas réservées aux ingénieurs supérieurs de l’Energie Atomique.

La réalisation la plus emblématique est le quartier des Escanaux où l'équipe Candilis/Josic/Woods propose une composition orthogonale articulant des immeubles bas de cinq niveaux à redents et des tours de quatorze étages. Deux lignes bordent la composition à partir desquelles se développent parallèlement ou perpendiculairement les immeubles bas. Cette disposition avec chaque ligne contenant trois tours, offre une échelle différente des « grands ensembles » rompant l'uniformité et donnant un caractère spécifique à chaque sous-ensemble, le centre étant occupé par le vide constitué par le stade et la piscine. 


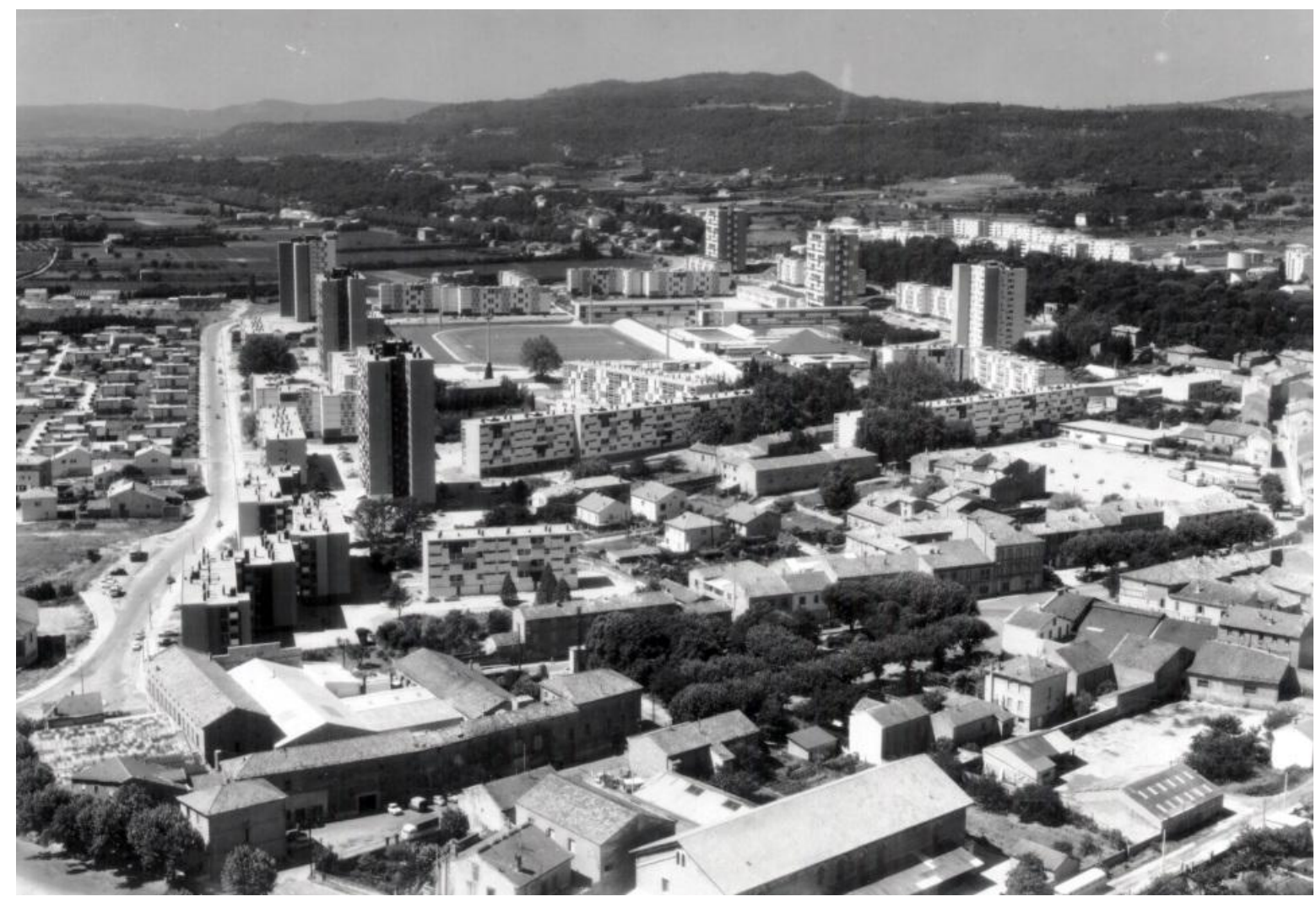

7. Les Escanaux à Bagnols sur Cèze, Candilis/Josic/Woods architectes, vue du ciel

La diversité des logements tente de transcrire dans des typologies modernes les qualités d'un habitat méditerranéen sur lequel Candilis et Woods ont déjà travaillé, notamment au Maroc, à Casablanca avec les immeubles Sémiramis et Nid d'Abeilles où l'espace du logement, principalement traversant, se prolonge à l'extérieur avec loggia, balcon ou terrasse séchoir permettant d'éclairer les salles de bains. Ainsi les immeubles de logements à cinq niveaux sont organisés par assemblage dont l'articulation est l'escalier autour duquel sont répartis symétriquement les logements avec des espaces communs réduits au minimum. Sur les façades de ces immeubles on observe une polychromie sur certains panneaux avec des couleurs primaires : bleu outremer, ocre rouge et ocre jaune.

Cette utilisation par panneaux monochromes rejoint sur certains points les recherches du groupe De Stijl ${ }^{34}$ et les compositions de Piet Mondrian mais aussi la polychromie architecturale de Le Corbusier ${ }^{35}$ expérimentée aux Quartier Modernes Frugès à Pessac.

Cette disposition qui offre de nombreuses variations de perspectives et de typologies de logements bénéficiant principalement d'une double orientation fait de cette opération un exemple qui obtiendra le premier grand prix national d'urbanisme en 1959.

Depuis sa construction le quartier des Escanaux a fait l'objet d'un certain nombre d'attentions. Ainsi des travaux de maintenance et d'entretien vont avoir des conséquences sur l'architecture comme l'isolation par l'extérieur des six tours en 1980. Une vêture est apposée sur les façades entrainant la perte de la force plastique et l'effacement des couleurs initiales, rendant les tours méconnaissables. Dix années plus tard, en 1990, est lancée une campagne de réhabilitation lourde avec changement des menuiseries extérieures en bois pour des

\footnotetext{
${ }^{34}$ Bois Yve-Alain and others: De Stijl et l'architecture en France, Bruxelles: Mardaga, 1985, p.16

${ }^{35}$ Colli Luisa Martina « Vers une polychromie architecturale »,1987 Le Corbusier une encyclopédie p.107
} 
menuiseries PVC accompagné d'un nivellement des façades par la suppression des balcons et séchoirs au profit de l'agrandissement des cuisines. Puis en 1998 des travaux sont engagés sur certains espaces extérieurs. Dans le même temps des aménagements sur les espaces extérieurs sont proposés dans un dessein analogue à celui de l'opération « Banlieues 89 » qui souhaitait consolider le lien entre centre ancien et ville nouvelle par une équipe dirigée par le paysagiste Alain Marguerit, sans résultat.

Le quartier s'inscrit également dans le cadre étatique de la politique de la ville depuis son classement en ZUS (zone urbaine sensible) en 1996. Ce classement s'est accompagné d'un contrat de ville signé en 2000 puis d'un contrat urbain de cohésion sociale (CUCS) en 2007. Plus récemment l'année 2009 a été l'occasion de célébrer par la ville l'anniversaire des 50 ans de sa construction et le quartier fait désormais partie des opérations du Programme National pour la Rénovation Urbaine (PNRU) depuis Décembre 2014.

Dans le même temps que l'extension de Bagnols sur Cèze l'équipe Candilis/Josic/Woods construira deux opérations à Nîmes, le Clos d'Orville et l'ensemble Tour de l'Évêque, (1958-1964), cette dernière définissant les fondements d'une typologie éponyme. Ces réalisations, avec celles du Blanc-Mesnil (1955-1957) et de Bobigny (1957-1962) «témoignent d'un intérêt nouveau pour la notion de continuité » ${ }^{36}$ et constitue une étape importante dans la réflexion de l'équipe Candilis-Josic-Woods sur la forme urbaine et les modes d'assemblages, amorçant les recherches ultérieures dans les réponses aux concours des années qui vont suivre.

\section{Georges Candilis et Team X}

La participation de Georges Candilis à la création de Team X trouve sa légitimité dans sa proximité avec Le Corbusier où dès 1949 il est chargé par ce dernier de le représenter au 7e CIAM. Membre de l'ASCORAL, de l'ATBAT dont il sera directeur de la filiale ATBAT Afrique, co-fondateur du groupe GAMMA, Candilis est en relation avec de nombreux acteurs des CIAM, et surtout présent dans les moments clés qui vont permettre l'élaboration de Team X comme la réunion des CIAM à Sigtuna en 1952 ou le congrès des CIAM d'Aix en Provence en 1953. Mais c'est lors de la réunion des CIAM à Londres en Août 1954 que Candilis s'exprime avec véhémence : «Nous pensons surtout après Aix que le moment est venu de donner de "l'eau de jouvence » au mouvement CIAM, de le réveiller et de l'orienter vers sa tradition et sa destinée - voie tracée par ses fondateurs, Corbu, Gropius, Giedion, Van Esteren, Sert, etc... Nous avons peur que : la sénilité, la fatigue, la médiocrité envahissent CIAM et que les congrès deviennent avec le temps un lieu de rencontres, de fêtes et d'admiration mutuelle, congrès identiques à tous les vétérinaires, des ichtyologues ou des amicales des anciens combattants, ni plus, ni moins. En examinant le congrès d'Aix, au point de vue congrès de travail et au point de vue résultats, il faut avoir le courage de dire la vérité. La façon de travailler par commissions, l'absence totale de préparation ou d'orientation du congrès, les résultats sont à tous points de vue plus que médiocres. Des choses mille fois répétées, des platitudes, des incompréhensions, l'esprit fade, des manques d'imagination et dilettantisme. Il faut rénover et nous voulons tenter notre chance ${ }^{37}$.Ces propos s'accompagnent d'une série de propositions en 8 points qui donnent de précises orientations sur l'organisation des futurs congrès notamment le CIAM X.

En décembre 1954 le désaccord entre les membres anglais (MARS) et hollandais (De 8 et Opbow) de Team X s'estompe notamment grâce à Bakema et une réunion du Team X se tient à l'agence de Candilis le 12 Avril 1955 tentant de redéfinir l'objet même des CIAM et leur futur. Quelques jours auparavant, le 8 Avril 1955 Le Corbusier dans une lettre au destinataire non identifié écrivait : «Les jeunes n'ont pas la force d'embrasser la

\footnotetext{
${ }^{36}$ Abram Joseph: L'architecture moderne en France, Paris: Picard, 2000 p.109

${ }^{37}$ Candilis Georges, Minutes de la réunion des CIAM, Londres 28-29 Août 1954, CIAM Special Collections, Frances Loeb Library / Harvard University GSD
} 
terrible complexité du phénomène moderne La largeur de vue prend peut être ici la forme d'absence de vue, ou alors adopter l'attitude des négations débilitantes et d'un dilettantisme verbal. La charte de l'habitat en tous cas devrait être la conclusion des 25 ans des CIAM et non pas le manifeste de la nouvelle étape. Les CIAM doivent léguer une charte et non pas en élaborer une. C'est très différent $»^{38}$

Etonnamment un mois après Le Corbusier change d'avis lors d'une réunion de préparation du congrès à venir avec Candilis et Bakema. Cette réunion, d'après Candilis, fut décisive pour Le Corbusier dans sa compréhension de l'agenda de Team X. Candilis pense que ce n'était pas avant ce jour-là que Le Corbusier avait saisi le thème du congrès proposé et que sous réserves de quelques confusions entre les nombreuses traductions anglaises et françaises il trouvait cela «très bien $»^{39}$ Quelques jours plus tard, fin Mai 1955 Le Corbusier et Candilis rédigent une invitation pour le $10 \mathrm{e}$ congrès qui doit se tenir à Alger ${ }^{40}$. Après de nombreuses vicissitudes sur le lieu du congrès, sa date et son contenu celui-ci se tient finalement à Dubrovnik du 3 au 13 Août 1956. Le Corbusier, qui n’y participera pas, envoie un message adressé au congrès dans lequel il insiste sur les différentes étapes des CIAM et où il précise qu'en 1956 «les CIAM-SECONDS» prennent le relais ${ }^{41}$

A Dubrovnik, Candilis est à la tête de la commission B7 sur l'urbanisme et l'habitat, tandis que la commission B6, « croissance et changement » est dirigée par Bakema et que la commission B4 sur la question du « cluster » est conduite par Peter Smithson. Le trio auquel il faut ajouter Aldo van Eyck, Alison Smithson et John Voelker vont se révéler les véritables artisans du passage des CIAM à Team X et les acteurs principaux des réunions à venir.

Le congrès d'Otterlo du 7 au 15 Septembre 1959 marque la transition entre les deux et c'est l'occasion pour Candilis de présenter le projet d'extension de Bagnols sur Cèze qu'il est en train de réaliser.

L'année d'après, en 1960, la ville de Bagnols sur Cèze sera le lieu de la première réunion de Team Ten programmée par Candilis. Les participants y débattent, s'y prennent en photo, envoient une carte postale à Le Corbusier et continuent les discussions entamées à Otterlo l'année précédente. C'est la première d'une série de 12 réunions dont Candilis en organisera quatre autres, à Paris en 1961, à Royaumont (avec Woods) en 1962, à Toulouse en 1971 puis la dernière à Bonnieux en 1977.

\footnotetext{
${ }^{38}$ Pedret Annie: Team X, an archival history, London - New York: Routledge, 2013 p.166 à 168

${ }^{39}$ Ibid

${ }^{40}$ Lettre du 23 Juillet 1956 de Le Corbusier adressé au Xe congrès des CIAM à Dubrovnik Special Collections, Frances Loeb Library / Harvard University GSD

${ }^{41}$ Pedret Annie: Team X, an archival history, London - New York: Routledge, 2013 p.180
} 


\begin{tabular}{l}
\hline Jaap Bakema (1914-1981) \\
\hline Aldo van Eyck (1918-1999) \\
\hline Georges Candilis (1913-1995) \\
\hline Giancarlo de Carlo (1919-2005) \\
\hline Peter Smithson (1923-2003) \\
\hline Alison Gill Smithson (1928-1993) \\
\hline Shadrach Woods (1923-1973) \\
\hline Bill Howell (1922-1974) \\
\hline Rolf Gutman (1926-2002) \\
\hline André Wogensky (1916-2004) \\
\hline John Voelker (1927-1972) \\
\hline Blanche Lemco (1923 \\
\hline Sandy Van Ginkel (1920-2009) \\
\hline Geir Grung (1926-1989) \\
\hline Jerzy Soltan (1913-2005) \\
\hline Josep Antoni Coderch (1913-1984) \\
\hline Ralph Erskine (1914-2005) \\
\hline Oskar Hansen (1922-2005) \\
\hline Karoly Polony (1928-2002) \\
\hline Louis Kahn (1901-1974) \\
\hline Kenzo Tange (1913-2005) \\
\hline Amancio Guedes (1925 \\
\hline Stephan Wewerka (1928-2013) \\
\hline Manfried Schiedhelm (1934-2011) \\
\hline Oswald M.Ungers (1926-2007) \\
\hline Guillermo Jullian de la Fuente (1931-2008) \\
\hline
\end{tabular}

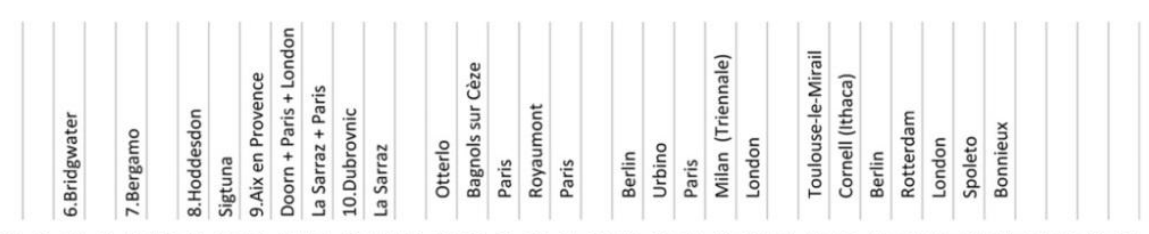

$4546474849505152535455565758596061626364 \quad 6566 \quad 6768 \quad 697071727374757677 \quad 787980 \quad 81$

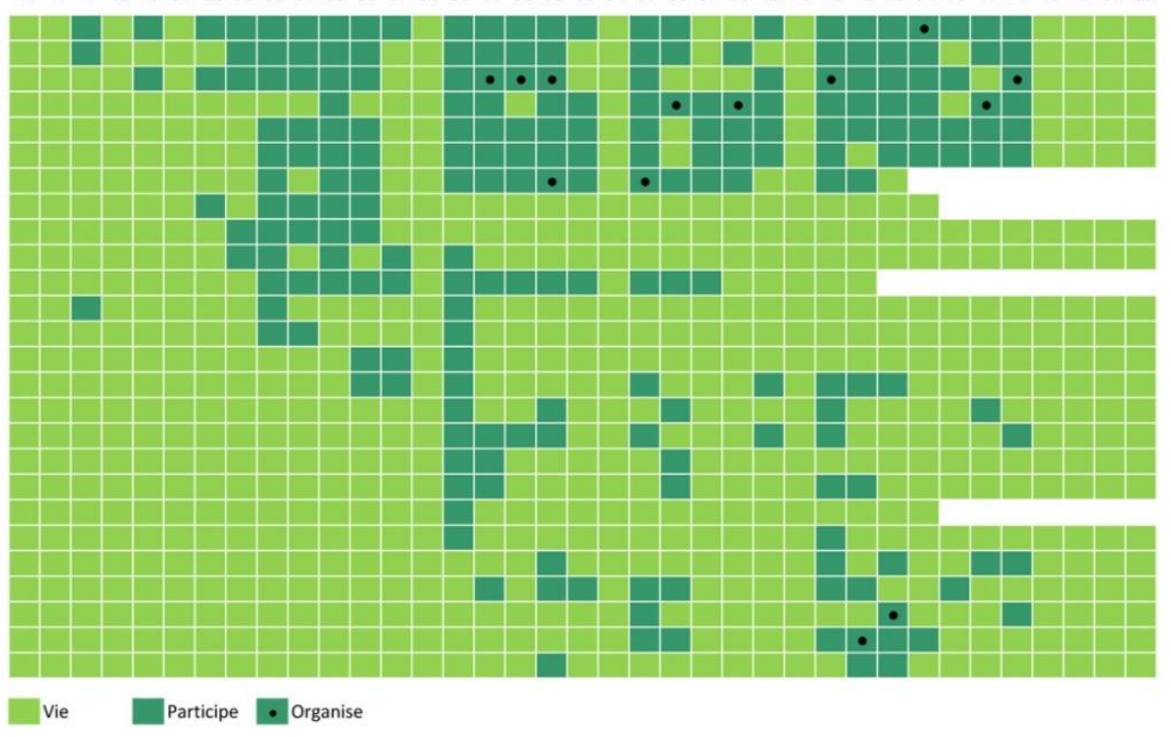

8. Les acteurs des CIAM (après 1945) et de Team X

Toujours en contact avec lui, Candilis informe Le Corbusier du programme de reconversion du Bas Rhône Languedoc $^{42}$. C'est l'occasion pour ce dernier de renouer avec Philippe Lamour, le président de la Compagnie nationale d'aménagement de la région du Bas-Rhône et du Languedoc et d'offrir ses services, sans résultat.

\subsection{Les concours}

Par la suite Georges Candilis et son équipe enchainent les concours : Francfort (1960), Caen-Hérouville puis Hambourg (1961). Ils sont lauréats en 1962 de la ZUP de Toulouse-Le Mirail devant Louis Arretche (2e) et Le Corbusier dont la proposition est classée 10e. Ce projet abandonne définitivement la notion de plan de masse au profit d'une autre manière de penser et de concevoir, s'appuyant sur les notions développées par Woods dans son article sur le «stem $»^{43}$.

La même année le concours pour l'université de Bochum puis celui pour l'université libre de Berlin (1963) sont l'occasion pour l'équipe de penser " l'université pour le plus grand nombre ». Les propositions démontrent encore de nouveaux systèmes d'organisation indépendants de la composition, basé sur le «...pendant et complément du stem, le web...structure horizontale en nappe qui forme une maille capable d'accueillir les activités et de les mettre en relation un réseau qui peut évoluer dans le temps aussi bien par extension externe que par transformation interne $»^{44}$.Ce projet fera l'objet d'un article par Alison Smithson intitulé : «How to

\footnotetext{
${ }^{42}$ Lettre de G.Candilis à Le Corbusier, 31 Octobre 1956 FLC H-3-20 : voir Ragot Gilles ; Dion Mathilde: Le Corbusier en France, Paris: Le Moniteur, 1987 p.374

${ }^{43}$ Shadrach Woods "Stem” Architectural Design n5 1960, p.61

44 Lucan Jacques: Composition, non-composition Architectures et Théories, XIXe-XXe siècles, Lausanne: Presses polytechniques et universitaires romandes, 2009 p.471
} 
recognize and read a mat building ${ }^{45}$ qui fera date dans l'histoire de l'architecture en le comparant au projet de Le Corbusier (+ Jullian) pour l'hôpital de Venise.

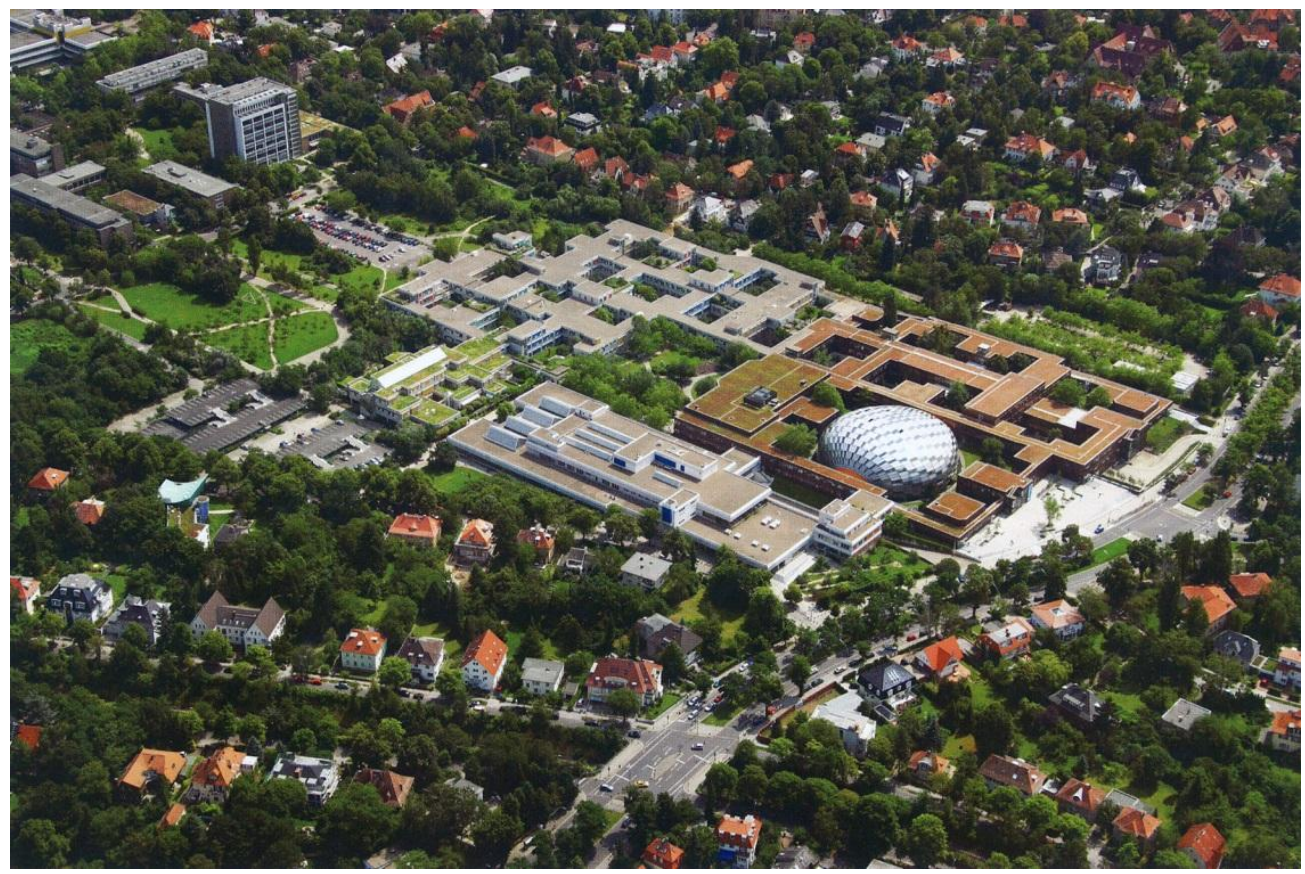

9. Vue aérienne Free University Berlin 2011, crédit photographique : Bavaria Luftbild/Freie Universität Berlin

Candilis réalisera également, pour partie, toute une série de projets d'universités appliquant le même principe typologique du « mat building » comme l'université de Toulouse le Mirail et de nombreuses autres qui ne seront finalement pas réalisées à l'instar de l'université de Zurich (1966), de Madrid (1969), de Larraquie (Syrie, 1973), Yarmouk (Jordanie, 1976) ou encore Hamadan (Iran, 1976).

S'en suivra une période où les projets s'enchaînent, tout comme les échelles. La conquête de nouveaux territoires viennent compléter cette expérimentation ainsi qu'un nouveau mode de vie : celui des loisirs. Ainsi Candilis participe à la mission Racine, mission interministérielle d'aménagement touristique du littoral du LanguedocRoussillon dont il assure la conception comme architecte en chef de la station de Leucate Barcares (1962-1976).

C'est la commande la plus importante que Candilis réalise sur le territoire français. Elle est constituée de plus d'une vingtaine d'opérations. Parmi elles, le village de vacances "Les Carats" est classé Monuments Historiques en 2014. De ce fait sont inscrits les façades, les toitures de tous les bâtiments et la totalité des bungalows de même que les parcelles et les aménagements situés sur la plage. Pour cette résidence, Candilis et Anja Blomsted conçoivent un mobilier en bois comprenant des chaises, tables, tabourets et lit/méridienne avec des équerres d'assemblage en aluminium ${ }^{46}$.

La conception de ce mobilier est sans aucun doute issue de sa passion pour le mobilier en bois courbé initiée par Le Corbusier et son attachement aux meubles Thonet comme le décrit Candilis : «Pendant toute sa vie, Le Corbusier dessine et meuble les intérieurs de ses édifices avec du Thonet. A tel point que certains s'imaginent qu'il en est le créateur ! ${ }^{47}$.

\footnotetext{
${ }^{45}$ Smithson Alison "How to recognize and read a mat building », Architectural Design Septembre 1974

${ }^{46}$ Voir le catalogue de l'exposition « Parallélisme géométrique » par Clément Cividino, Fondation Vasarély, Février 2015

${ }^{47}$ Candilis Georges: Meubles en bois courbé, Stuttgart - Zurich: Krämer Verlag, 1980 p.7
} 

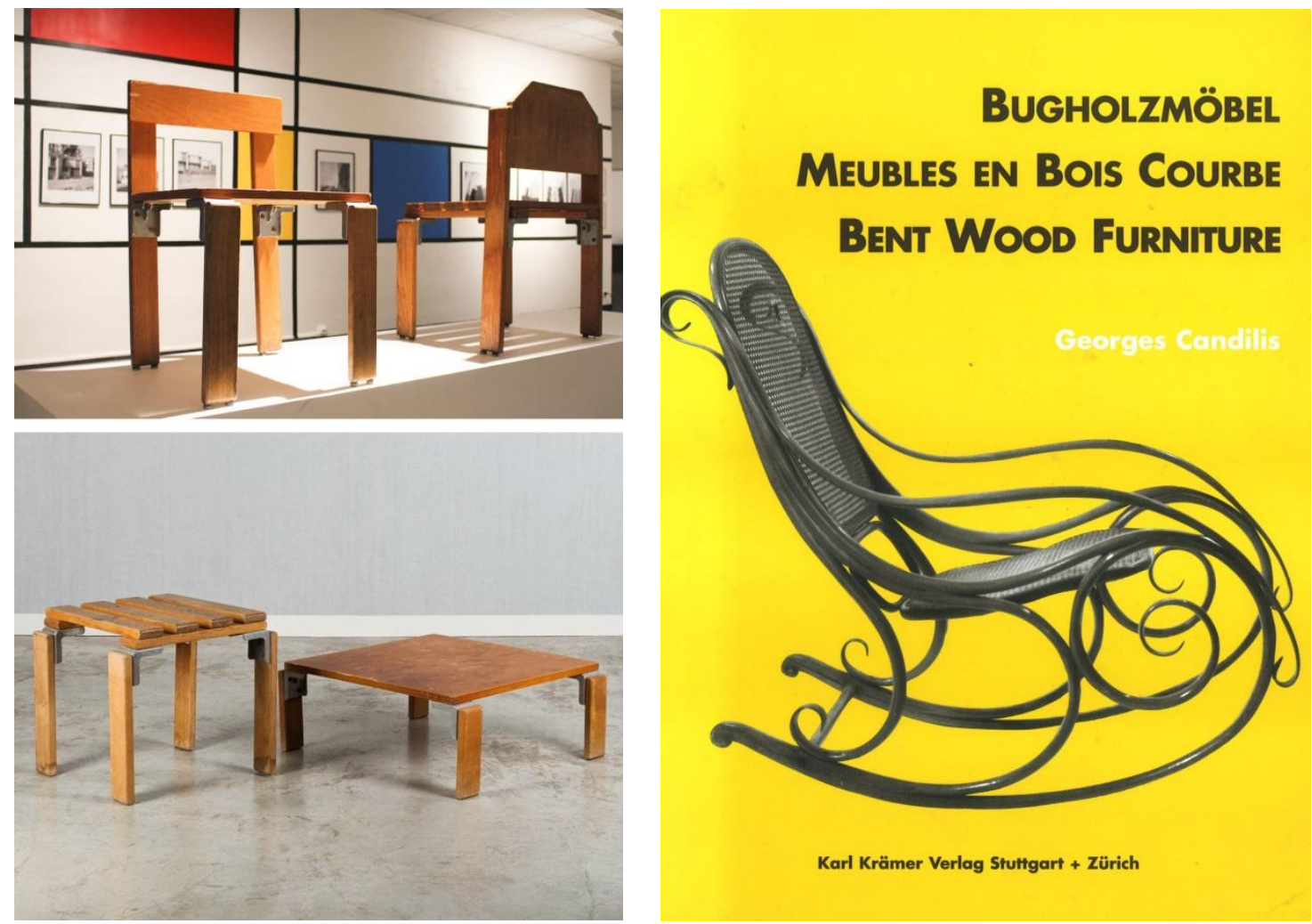

10. Meubles en bois de Georges Candilis et Anja Blomsted,

11. Couverture du livre de Georges Candilis, Meubles en bois courbé

En 1972, dans ce territoire qu'était le Languedoc Roussillon, sorte de laboratoire pour les loisirs, Georges Candilis lance la formule du « camping de l'an 2000 » en implantant à titre expérimental 27 hexacubes sur la commune de Leucate. Conçu par Georges Candilis et Anja Blomsted, l'hexacube ${ }^{48}$ est un nouveau procédé d'habitat modulaire composé de coques en polyester assemblées, constituant des modules habitables de 8 à $12 \mathrm{~m}^{2}$ groupables et permettant de nombreuses combinaisons.

\footnotetext{
${ }^{48}$ Fond Candilis CAP Hexacube / le cubing : tourisme-loisirs-travail CANGE/H/ND/15
} 


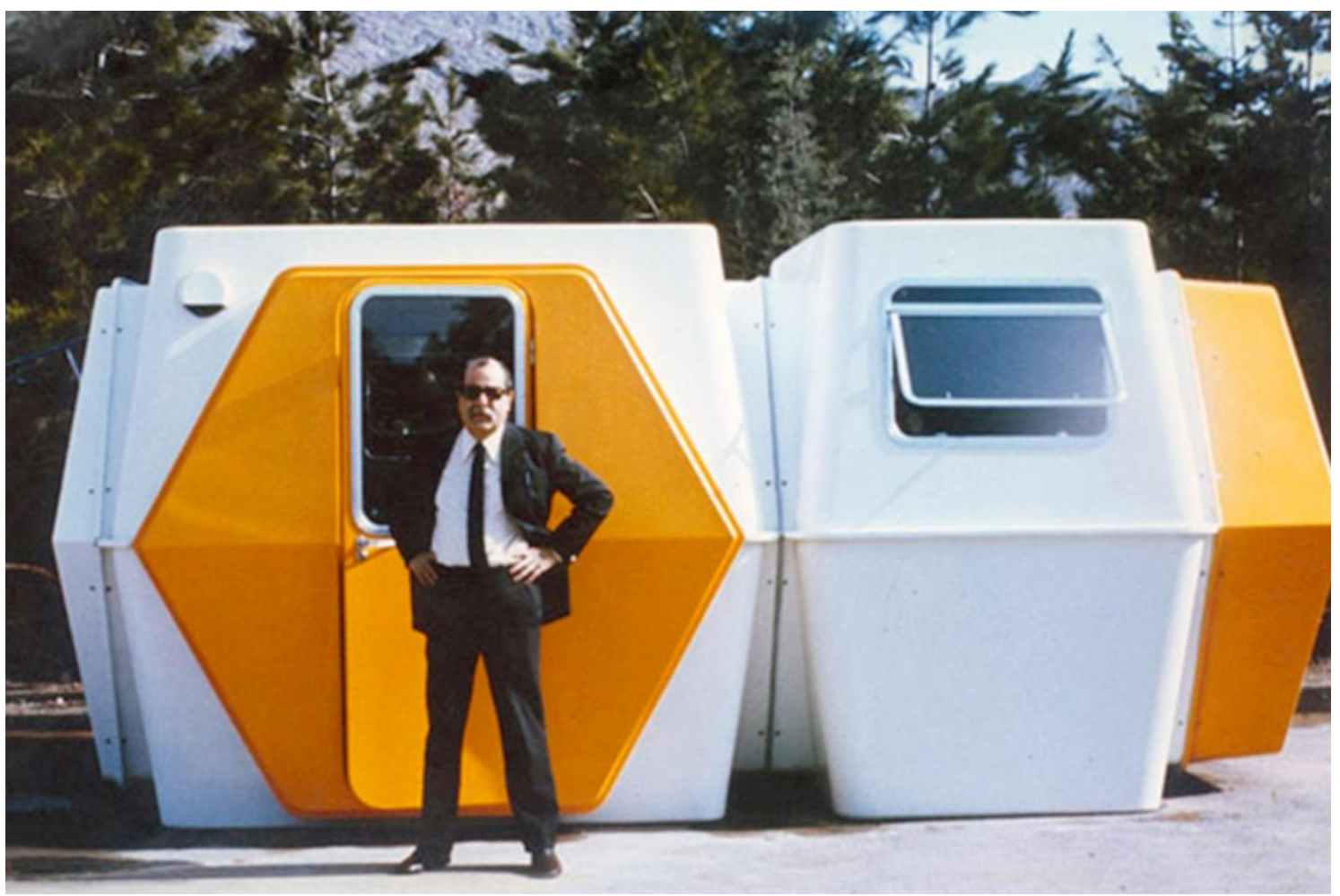

12. Georges Candilis devant l'Hexacube

Entre 1970 et 1978 Candilis est chargé de plusieurs projets au Moyen Orient. De nombreux ensembles résidentiels et d'habitation sont étudiés en Iran, au Koweït, au Qatar et en Arabie Saoudite, certains seront réalisés témoignant ainsi d'une production de Candilis toujours grandissante. Au milieu des années 70, en plus des agences de la rue Dauphine, de Toulouse, de Berlin et d'Athènes, Candilis dirige d'autres structures au Liban et en Arabie Saoudite, l'ensemble totalisant plus de quatre-vingts personnes.

En 1979, de retour en Grèce Georges Candilis « en tant que responsable du développement du Centre Culturel d'Athènes devient président de la délégation grecque à l'Unesco, du Centre International de Delphes et responsable de la candidature Grecque aux Jeux Olympiques de 1996. Juste avant sa mort, en 1995 il livre un très important travail de réflexion sur le futur des J.O. au seuil du XXIe siècle ${ }^{49}$.

\section{Diffusion et transmission}

Candilis, s'il ne se révèle pas comme un grand théoricien, a toujours habilement diffusé ses idées et communiqué sur ses réalisations. Souvent acteur au sein d'un groupe (CIAM, ASCORAL, ATBAT, GAMMA, TEAM X...) il est surtout membre du comité de rédaction de l'Architecture d'Aujourd'hui dès 1953 et ce durant 20 ans. La revue est une véritable tribune pour la publication de ses réalisations dès le numéro 46 daté de 1953. Candilis publie également des articles qui se rapportent à sa pratique ou qui l'explicitent. Les plus importants sont «L'esprit du plan de masse de l'habitat» paru dans l'Architecture d'Aujourd'hui n ${ }^{\circ} 57$ de 1954 mais surtout l'article intitulé «A la recherche d'un sens nouveau du mot architecte» paru en $1975:$ : La responsabilité et le rôle de l'architecte parmi les gens qui prennent les décisions pour l'avenir deviennent de plus en plus importantes. Jamais, dans l'évolution de l'humanité, sa présence n'a été aussi nécessaire. Depuis le

\footnotetext{
49 Demetriades Dimitri \& Papadaniel Dimitri, Entretiens avec Georges Candilis, Article de la revue des Ingénieurs et architectes suisses, 1994
} 
commencement de notre siècle, l'apparition de très timide de la notion dite "urbanisme" domine de plus en plus la vie de la société. L'architecture et l'urbanisme se confondent aujourd'hui en une seule discipline, celle de "l'art de bâtir", action étroitement liés à la condition et à la possibilité pour l'homme d'agir, de penser et d'aimer. L'acte de bâtir ne peut plus être pris comme un acte isolé, privilège exclusif d'une profession ou d'un corps constitué. C'est une action collective qui concerne tout le monde, se confond avec la vie même» ${ }^{50}$

Candilis publie également de nombreux articles sur l'architecture en général et sa diffusion mais aussi sur le rôle de l'architecte dans Techniques et Architecture, le Carré bleu, Architectural Design, Architecture Formes Fonctions, Bauwelt, Bauen + Wohnen ce qui lui confère une audience qui dépasse largement les frontières de l'hexagone.

De même concernant l'enseignement de l'architecture Candilis s'y implique fortement jusqu'au début des années 80 où il est alors professeur à l'UP6. Ainsi dès 1963 Candilis et Josic fondent l'atelier extérieur Candilis-Josic, les étudiants se retrouvant régulièrement dans les locaux de l'équipe rue Dauphine. L'année 1965 est l'occasion pour Candilis de commencer à enseigner à la tête de l'un des six ateliers (au sein du groupe C) de l'Ecole Nationale Supérieure des Beaux-Arts qui s'installent au Grand Palais. Sa création est un événement majeur dans l'École des beaux-arts de l'avant Mai 68.

Candilis va rédiger quelques pages où il indique «Aux matières scientifiques complémentaires exigées dans l'enseignement de l'Architecture : les mathématiques, la physique, la chimie, le génie civil, la statique et la mécanique théorique, s'ajoutent aujourd'hui au même titre, et de même importance la sociologie et l'économie politique, la psychologie et la logique, l'histoire et la géographie appliquées, la biologie, le droit et la philosophie... Une cuvre bâtie, une construction, devient "Architecture » quand elle exprime avant tout l'essence de son temps » et de conclure : «A l'enseignement d'hier qui avait pour base : apprendre comment il faut construire, doit s'ajouter un enseignement plus ouvert qui doit aider à concevoir ce qu'il faut construire, car demain il ne suffira plus simplement de connaître mais il faudra comprendre, imaginer et créer un monde nouveau, meilleur que celui d'hier, et pour cela nous devons pouvoir aller jusqu'au cour de la vérité» ${ }^{51}$ :

\section{Acknowledgements}

Ines Zalduendo, Special Collections, Frances Loeb Library / Harvard University GSD

Shelley Hayreh, Avery Architectural and Fine arts Library, Columbia University

\section{Sources of Images :}

Image 1 : Dépliant de présentation pour le 7e congrès CIAM à Bergame, Special Collections, Frances Loeb Library / Harvard University GSD

Image 2 : Immeuble à Sidi Bel Abbès, Candilis \& Woods architectes, 1954 Woods Archives, Avery Architectural and Fine arts Library, Columbia University

Image 3 : Candilis Georges \& Woods Shadrach photographie dessin sur calque Habitation collective marocaine, recherches sur la trame Modulor, Déc.1951, Woods Archives, Avery Architectural and Fine arts Library, Columbia University

\footnotetext{
${ }^{50}$ Candilis Georges « A la recherche d'un sens nouveau au mot architecte » Architecture Formes Fonctions n ${ }^{\circ} 151969$ p.1321, texte repris et complété dans l'Architecture d'Aujourd'hui n 177 « TEAM $10+20$ », Février 1975 p. 50 \& 51

${ }^{51}$ Candilis Georges « Schéma pour une orientation vers la réforme de l'enseignement de l'architecture » Avril 1965, Texte non publié, Fonds Candilis CAP CANG 318/01
} 
Image 4 : Revue СОВРЕМЕННАЯ АРХИТЕКТҮРА, l’Architecture contemporaine $\mathrm{n}^{\circ} 4$ \& 5 , archives Famille Candilis

Image 5 : Immeuble Sémiramis, Carrières Centrales, Casablanca, Candilis \& Woods architectes, Woods Archives, Avery Library, Columbia University

Image 6 : croquis et schémas d'intention, extension de Bagnols sur Cèze, Candilis/Josic/Woods Woods Archives, Avery Architectural and Fine arts Library, Columbia University

Image 7: Les Escanaux à Bagnols sur Cèze, Candilis/Josic/Woods architectes, vue du ciel, tirée de La Construction Moderne Française, fascicule, 1965, CAP Fonds Candilis

Image 8 : les acteurs des CIAM (après 1945) et de Team X

Image 9 : Vue aérienne Free University Berlin tirée de University Planning and Architecture, The Search for perfection, 2011, crédit photographique : Bavaria Luftbild/Freie Universität Berlin

Image 10 : Meubles en bois de Georges Candilis et Anja Blomsted, exposition «Parallélisme géométrique » par Clément Cividino, Fondation Vasarély, Février 2015

Image 11 : Couverture du livre de Georges Candilis, Meubles en bois courbé, Stuttgart - Zurich: Krämer Verlag, 1980 ,

Image 12 : Georges Candilis devant l'hexacube Fond Candilis CAP CANGE/H/ND/15

\section{Bibliography / references}

Abram Joseph: L'architecture moderne en France, Paris: Picard, 2000

Bois Yve-Alain and others: De Stijl et l'architecture en France, Bruxelles: Mardaga, 1985

Candilis Georges, Josic Alexis, Woods Shadrach: Proposition pour un habitat évolutif, Techniques et Architecture ${ }^{\circ} 2,1959$

Candilis Georges: A la recherche d'un sens nouveau au mot architecte, Architecture Formes Fonctions $\mathrm{n}^{\circ} 15$ 1969

Candilis Georges: A la recherche d'un sens nouveau au mot architecte, l'Architecture d'Aujourd'hui n 177 «TEAM $10+20 »$, Février 1975

Candilis Georges: Bâtir la vie, un architecte témoin de son temps, Paris: Stock, 1977

Candilis Georges: Meubles en bois courbé, Stuttgart - Zurich: Krämer Verlag, 1980

Cohen Jean-Louis ; Eleb Monique: Casablanca : Mythes et figures d'une aventure urbaine, Paris: Hazan, 1998

Coulson Jonathan; Roberts Paul; Taylor Isabelle: University Planning and Architecture, The Search for perfection, London - New York: Routledge, 2011

Demetriades Dimitri \& Papadaniel Dimitri: Entretiens avec Georges Candilis, Revue des Ingénieurs et architectes suisses, 1994

Lucan Jacques: Composition, non-composition Architectures et Théories, XIXe-XXe siècles, Lausanne: Presses polytechniques et universitaires romandes, 2009

Mumford Eric: The CIAM discourse on urbanism 1928-1960, Cambridge: MIT Press, 2000

Pedret Annie: Team X, an archival history, London - New York: Routledge, 2013

Ragot Gilles ; Dion Mathilde: Le Corbusier en France , Paris: Le Moniteur, 1987

Sarkis Hashim: CASE: Le Corbusier's Venice Hospital and the Mat Building Revival, Munich-London-New York: Prestel Verlag, 2001

Smithson Alison \& Peter: Collective Housing in Morocco" Architectural Design, janvier 1955 
Smithson Alison: How to recognize and read a mat building », Architectural Design Septembre 1974

Woods Shadrach: Stem Architectural Design n5 1960

\section{Documents :}

\section{Fonds CAP CANDILIS Georges (1913-1995): CANGE}

Candilis Georges «Schéma pour une orientation vers la réforme de l'enseignement de l'architecture » Avril 1965, Texte non publié, Fonds Candilis CAP CANG 318/01

La Construction Moderne Française, fascicule, 1965

L'Hexacube / le cubing : tourisme-loisirs-travail CANGE/H/ND/15

\section{Woods Archives, Avery Architectural and Fine arts Library, Columbia University}

Photographie Immeuble à Sidi Bel Abbès, Candilis \& Woods architectes, 1954

Photographie dessin sur calque Habitation collective marocaine, recherches sur la trame Modulor, Déc.1951

Photographie Immeuble Sémiramis, Carrières Centrales, Casablanca, Candilis \& Woods architectes

Croquis et schémas d'intention, extension de Bagnols sur Cèze, Candilis/Josic/Woods architectes

Special Collections, Frances Loeb Library / Harvard University GSD

Lettre du 23 Juillet 1956 de Le Corbusier adressé au Xe congrès des CIAM à Dubrovnik CIAM Special Collections

Dépliant de présentation de l'Unité d'Habitation de Marseille pour le $7^{\mathrm{e}}$ congrès CIAM à Bergame

Candilis Georges, Minutes de la réunion des CIAM, Londres 28-29 Août 1954, CIAM Special Collections

\section{$\underline{\text { Archives Famille Candilis }}$}

Revue СОВРЕМЕННАЯ АРХИТЕКТҮРА, l'Architecture contemporaine $n^{\circ} 4 \& 5$

Lettre de Le Corbusier à Candilis datée du 24 Mai 1949 